\title{
How the Utilisation of 3D Body Scanners Enhance the Construction of Traditional Sawwan Garments
}

\author{
Faizah ALMALKI *, Simeon GILL, Steven G. HAYES, Lisa TAYLOR \\ The University of Manchester, Manchester, UK
}

https://doi.org/10.15221/21.49

\begin{abstract}
The garment industry relies upon direct measurements of the human form to ensure that items of clothing fit as well as possible. Over time, conventional measurements taken using manual methods have been replaced and enhanced by the output of 3D digital body scanners. This study identifies the specific measurements produced by 3D body scanning and compares these with the measurements produced using conventional methods. This data is then used to improve the creation of a pattern for the traditional Sawwan garment that part of the cultural identity of a region of Saudi Arabia. Traditional clothing such as this provides insight into the heritage of a region and adopting new technology in its manufacture sustains access to traditional garments. This study considers how the utilisation of 3D body scanners rather than conventional, manual measuring methods can enhance the construction of Sawwan garments for individuals. Importantly, the greater depth of data afforded by 3D body scanners than manual methods when measuring the human form creates opportunities for garments to be tailored digitally. Consequently, there is a need for the measurement profile to define the required measurements relating to the basic block construction. For the purpose of this research, a widelyutilised garment draft is compared to a new novel method using CAD pattern software (Lectra Modaris Expert) which utilises the measurements afforded by a 3D body scanner. As such, there is a need for highly accurate and consistent measurements in order for a garment to offer a good fit. In addition, it is anticipated that this study will prompt future researchers to investigate the potential for modern technology to help produce traditional garments and enhance our grasp of pattern theory. It is apparent from the findings of the current study that 3D body scanners offer a greater number of highly accurate measurements than would be possible when relying on conventional methods and this can help to improve how Sawwan garments are produced. The findings help to enhance our appreciation of the contribution that $3 \mathrm{D}$ body scanners can make to patternmaking practice and its evolution. It helps to further the development of technology-assisted pattern drafting and expands the variety of garments accessible to consumers in Saudi Arabia, including traditional garments often made by hand.
\end{abstract}

Keywords: 3D body scanning, Pattern Cutting, Bespoke Pattern Cutting, Traditional Garments

\section{Introduction and background}

The current research sets out to explore the Sawwan (1958) which is an item of clothing that the women of Bani Malik in Saudi Arabia would traditionally wear (see Figure1). Written instructions for how to construct a Sawwan are unavailable and, therefore, it is necessary to resort to illustrations of the garments as well as people's testimonies to appreciate how such items are constructed $[1,2,3,4,5]$. When attempting to produce a garment that fits well, it is necessary to take measurements directly from the intended wearer. Until very recently, such measurements would need to be taken manually but it is becoming increasingly common for individuals to be measured using a 3D digital body scanner. The current study explores the various measurements that can be taken using a 3D body scanner and makes comparisons with the conventional manual measurements. In turn, this insight is utilised to enhance patternmaking methods for the Sawwan garment which is part of Saudi regional culture. The Sawwan is emblematic of regional heritage and by applying the latest technology for the purpose of manufacturing, this will help to ensure as many people as possible are able to continue enjoying such garments. More specifically, the current study gives thought to how the adoption of 3D body scanners for the purpose of measuring individuals can improve the construction process of patterns and garments and benefit the wearer. 




Figure 1 traditional Sawwan garment that worn by women in Bani Malik, 1958

Owing to the fact that 3D body scanners are able to generate a much greater depth of data relative to conventional methods of measuring, the new technology opens up the potential for the digital tailoring of garments. For this opportunity to be realised, however, there must be a measurement profile so that the measurements necessary for the block construction can be defined. In the current study, comparisons are made between a traditional garment draft and a modern approach whereby measurements obtained from a 3D body scanner are applied to the Lectra Modaris Expert CAD pattern software. Measurements generated by body scanners create the possibility to enhance the drafting methods for pattern blocks (slopers) [6]. Body scanners are capable of generating significantly more measurements than would be possible when relying on conventional means of measuring [7]. As such, the opportunities for 3D body scanners to be applied in the garment sector are undeniable. In only a matter of seconds, 3D body scanners can generate a comprehensive set of measurements of the human form [8]. Importantly, because body scanners also generate a depiction of the body in image form (a digital replica), changes can easily be made to the measurements being taken if necessary. Crucially, body scanners offer advantages in terms of the time taken to measure the human form but also the accuracy and quantity of those measurements.

It is widely recognised that the measurements taken by a 3D body scanner will be no less accurate than those taken by manual means [9]. Crucially, there is the potential to produce garments that fit the wearer very well owing to the ability to take a large number of linear and non-linear measurements. Moreover, the measurements taken by body scanners are stored digitally and this creates an opportunity for scanners to be directly linked to CAD systems which would not only save time but also reduce the potential for human error. Therefore, body scanners open up the possibility of mass producing customised items of clothing. The current research draws upon a traditional style and traditional methods of draping but utilises a greatly increased number of measurements that is only possible because of the modern body scanning technology. As such, the pattern blocks created will better reflect the proportions, size and shape of the wearer. Therefore, the current study not only offers a demonstration of how body scanners can help to develop patterns but also the variety of measurements that can be generated. 
Consequently, body scanning technology will enable manufacturers to create garments that are not only more functional but also offer the wearer a much improved fit. In terms of the Sawwan, this research provides a clear demonstration of how modern technology can be applied alongside conventional means of pattern cutting to create a new opportunity for clothing manufacturers to exploit. Traditionally, Sawwan garments would be produced without any attempt to measure the hips of bust of the intended wearer. Therefore, the current study makes a valuable contribution to the existing body of knowledge, using modern technology to significantly enhance patternmaking methods. Owing to the fact that almost the entire process is automated, customised patterns can be generated whilst also offering customers greater choice in terms of the styles of garments available. This should help to encourage people to wear functional interpretations of traditional styles on a daily basis, thereby preserving regional customs for future generations to enjoy.

\section{Method}

In order to realise the stated aims of the current study, the following methods will be applied:

\section{Process for the proposed Sawwan pattern method using 3D body scan}

It is possible for garments to be customised with insight into the nature of the relationship between the human form and patterns shapes [10]. Once a pattern block has been produced for a particular item of clothing, it can be amended so that the resulting garment is tailored to fit [11]. This section sets out the selected approach for organising and managing the data collection process and sample preparation. A Size Stream (SS) body scanner (SS20) and software (SSStudio 5.2.9) was employed to generate the necessary quantitative measurements. The initial body selection was a size 12, $\mathrm{M}$ and 24 Alvanon dress form. Rather than studying actual people, the decision was taken to utilise body forms owing to the fact that they are symmetrical and offer measurement landmarks that are stable [12]. As part of the process, it was necessary for any errors identified with the draft to be amended with corrections being signed off and care being exercised to ensure accuracy. The use of dress forms helps to ensure stability because they assist in efforts to identify crucial elements of the adopted method. The research set out to explore the processes involved in pattern cutting whilst referring to previous studies that have made use of body scanning technology.

\section{Testing the new method of Sawwan patternmaking}

Following testing on the dress forms a total of five healthy adults participated in actual fittings; one was size small, one was medium, one was large and two were extra-large, albeit they had different body configurations. Before any measurements were taken manually, care was taken to identify the necessary landmarks. Having referred to a table they had received from the researcher, the participants took manual measurements using a tape measure and these were recorded in centimetres. The same seventeen measurements were taken by the body scanner and logged in the table: length of sleeve, wrist, length of pattern, depth of armscye, waist to nape, width of back, depth of the back of the neck, width of the back of the neck, width of the front of the neck, depth of the front of the neck, hip to waist, length of bust, length of sleeve, length of back, shoulder, hips, circumference of the waist, circumference of the bust. The adopted methods derived from ISO 7250 and ISO 8559. Measurements were taken with the participants wearing only their underwear whilst standing. Once the measurements had been recorded, CAD software was used to draft individual patterns for each of the participants. Once the garments had been produced from the bespoke patterns, the participants tried them on, gave their assessment of how well they fitted and how comfortable they were and photographed themselves. The researcher received a copy of the photographs with the participants' faces obscured. The focus of the images was on the item of clothing and the extent to which the pattern had been determined by the human form. The current study sought to determine whether manual methods could replicate the accuracy of the measurements derived from a body scanner. From the results, it is apparent that the participants were able to take a total of twelve measurements: length of the sleeve, wrist, length of the pattern, depth of the armscye, waist to nape, width of the back, length of the bust, length of the back, shoulder, hips, circumference of the waist, circumference of the bust. The following table provides details of the measurements and the associated definition as set out in ISO 8559: 2017. 


\begin{tabular}{|c|c|c|}
\hline ISO 8559 ID & Measurements & Definition \\
\hline 5.3 .4 & Bust girth & Horizontal girth measured at a bust point level \\
\hline 5.3 .10 & Waist girth & The horizontal girth of the body measured at the waist level \\
\hline 5.3 .13 & Hip girth & The horizontal girth of the body measured at the hip level \\
\hline 5.3 .19 & Wrist girth & The girth of the wrist at the level of wrist point \\
\hline 5.4 .1 & Shoulder length & Distance from the side neck point to the shoulder point \\
\hline 5.4 .5 & back length to waist & Distance from the back neck point to waist level \\
\hline 5.4 .21 & Side waist to hip & $\begin{array}{l}\text { The distance down the side of the body from the waist level to } \\
\text { the hip level }\end{array}$ \\
\hline 5.4 .11 & $\begin{array}{l}\text { Side neck point to waist } \\
\text { level }\end{array}$ & $\begin{array}{l}\text { Distance from side neck point, over the bust point, then vertically } \\
\text { straight to the waist level }\end{array}$ \\
\hline 5.4 .6 & Scye depth length & $\begin{array}{l}\text { The distance measured vertically on the body from the back } \\
\text { neck point to the upper edge of a tape measure passing } \\
\text { horizontally under the arms at the axilla }\end{array}$ \\
\hline 5.1 .5 & Back neck height & The vertical distance from the back neck point to the ground \\
\hline 5.3 .3 & Neck base girth & $\begin{array}{l}\text { The girth of the base of the neck measured over the back neck } \\
\text { point and the front neck point }\end{array}$ \\
\hline
\end{tabular}

\section{Results and discussion}

Utilising a body scanner creates the possibility of generating patterns that better reflect the true proportions, shape and size of the wearer. Measurements that are not easily obtained by using a tape measure have traditionally been estimated based on a proportional rule but this can result in the pattern diverging significantly from the true body measurements. The armhole depth provides a good example of how the pattern can be more closely related to the actual body of the wearer. The depth of the armhole is typically interpreted as the length along the surface from the centre of the spine at the back of the neck to a point level with the lowest armpit. In the absence of tools, this measurement can be difficult to take accurately. Not only is the body scanner able to measure this length highly accurately but it is also able to take the shoulder blade into consideration. 
Table 1 The following table shows the additional measurements that were taken from the $3 D$ scanner, while the manual method was unable to take them.
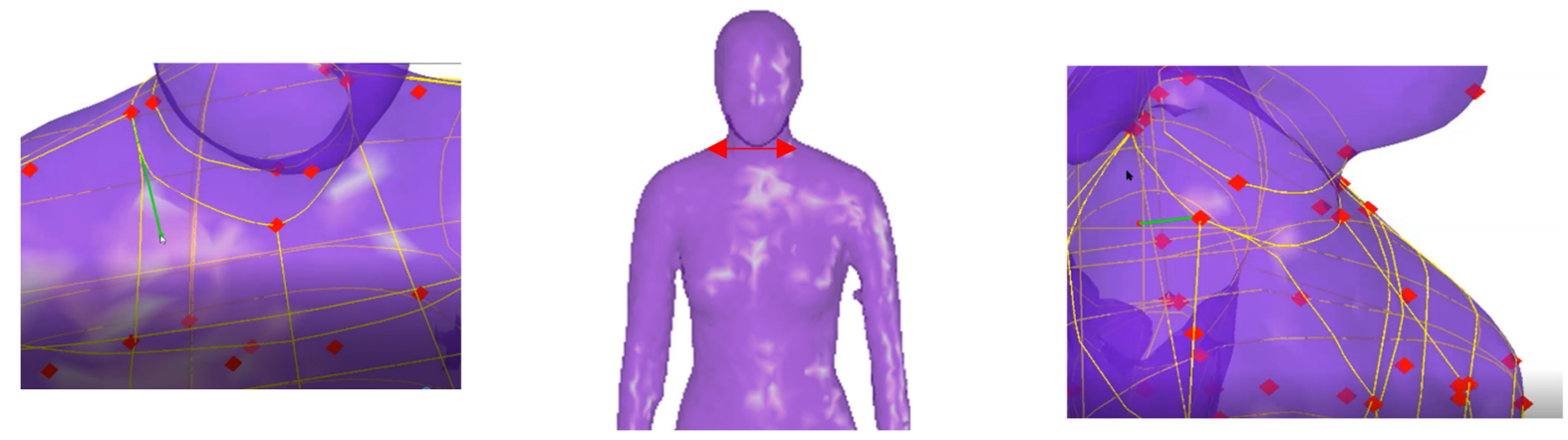

Front neck depth :Measure the vertical measurement from the centre neck level to the neck points.

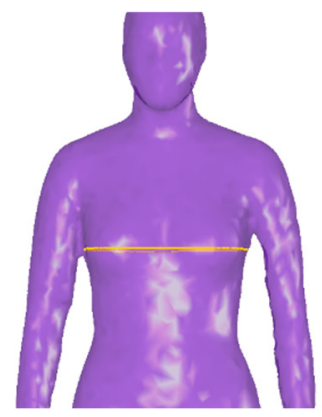

Fr Bust Arc: scan the bust circumference to measure the fullest point of the bust from the front and back in the loop line. Therefore, that could be divided into ARC (Bust Front Arc \& Bus Back t Arc).



hip Arc: 3D body scans a vertical measurement around the hip and defines the right position for the hip point.
Front neck width: Neck width can be extracted directly from distance between the side neck points.

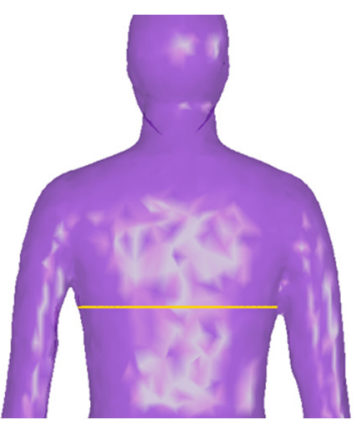

Bk Bust Arc: scan the bust circumference to measure the fullest point of the bust from the front and back in the loop line. Therefore, that could be divided into ARC (Bust Front Arc \& Bus Back t Arc).
Back neck depth: It is a difference between the side neck and back neck

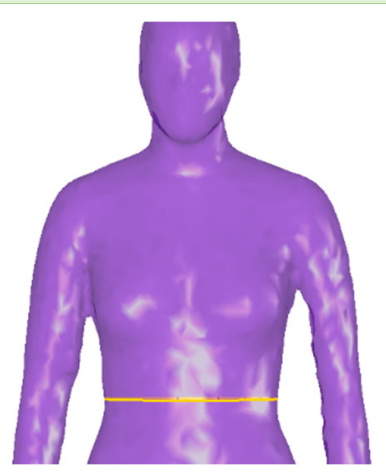

Waist Arc: 3D body scans a vertical measurement around the waist and defines the right position for the waist point.

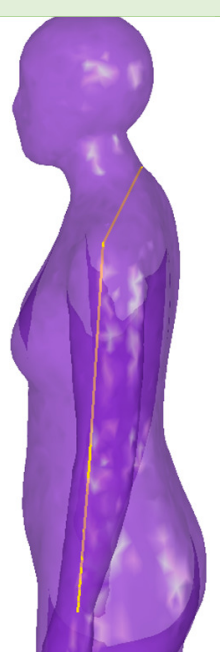

Centre back neck to centre back chest: This measurement defines the depth of the armhole. However, starting from the neck bone down to the centre back chest.

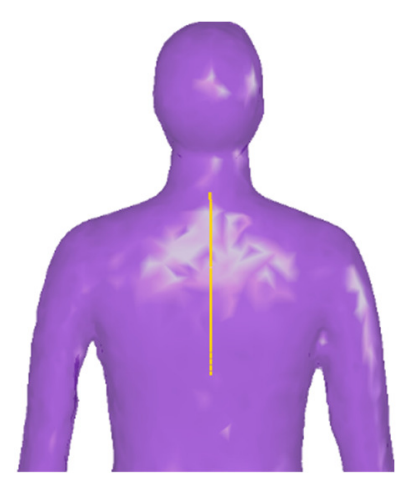

sleeve length: In 3Dbody scan, the hand is relaxed. Then take a measurement starting at neck bone passing over the shoulder bone and the elbow to the wrist bone. 


\section{Conclusion}

It is apparent that 3D body scanning technology offers the ability to take reliable measurements of the human form that can enhance the process of patternmaking. Be that as it may, it will only be possible to truly realise the full potential afforded by body scanners if the process can be linked directly to pattern cutting and fully automated. Analysis systems must now be devised that make use of all available measurements to improve patternmaking. This will facilitate the assimilation of systems into the traditional methods that are applied at present. It is necessary to map the process to align the body scanner outputs to those required to create the bespoke pattern draft.

Such research is necessary to provide a better grasp of pattern theory and the way in which measurements are used to produce a pattern shape. In addition, research is required to establish the most appropriate place to site the waist and bust darts. It may be possible to arrive at the optimal dart length if suitable analysis tools were to be developed. Similarly, the most appropriate dart openings could be confirmed based on analysis of arcs, depths and widths, data which can be derived more easily from body scanning.

Body scanners take measurements of the human form in a particularly efficient way and the results can be utilised when making patterns. Importantly, effective programming can result in the entire process being effectively automated, thereby transforming the means of producing pattern blocks so that madeto-measure clothing can be mass produced.

\section{Acknowledgements}

I would like to express my sincere gratitude to Al Bahah University for making this research possible which has given me a new skillset that will be passed on to future generations.

\section{References}

[1] Mimny, E. (1996) Study the Development of Traditional Clothing inherited and Accessorise for Saudi Women in Taif Region. Umm AlQura University, Meccah, The Kingdom of Saudi Arabia.

[2] Almalki, F. (2017) Remaking the Past: Fashion Heritage Futures in Bani Malik Al Hijaz, Saudi Arabia. Master Thesis, University of Wolverhampton.

[3] Albasam, L. (1985) Traditional heritage of women's clothing in Najd. (1st ed). Qatar: Center for Folklore of the Arab Gulf States.

[4] Ajaji, T. (2005) Traditional Women's Costumes in the northern region of Saudi Arabia. College of Education for Home Economics, Riyadh, Saudi Arabia.

[5] Iskandarani, B. (2006) Traditional Clothing for Women and Bride Clothes in Medina. Scientific algorithm for publication and distribution

[6] Gill, S. et al. (2018) 'Scan to Pattern: How Body Scanning Can Help Transform Traditional Methods of Creating Pattern Blocks', pp. 16-17. doi: 10.15221/18.236.

[7] E. Bye, K. L. Labat, and M. R. Delong, "Analysis of Body Measurement Systems for Apparel," Cloth. Text. Res. J., vol. 24, no. 2, pp. 66-79, Mar. 2006.

[8] Istook, C. L. and Hwang, S. J. (2001) '3D body scanning systems with application to the apparel industry', Journal of Fashion Marketing and Management, 5(2), pp. 120-132. doi: 10.1108/EUM0000000007283

[9] Wang, Y. J. et al. (2011) 'Body measurements of Chinese males in dynamic postures and application', Applied Ergonomics, 42(6), pp. 900-912. doi: 10.1016/j.apergo.2011.02.006.

[10] McKinney, E. et al. (2017) 'Body-to-Pattern Relationships in Women's Trouser Drafting Methods: Implications for Apparel Mass Customization', Clothing and Textiles Research Journal, 35(1), pp. 16-32. doi: 10.1177/0887302X16664406.

[11] Gill, S. and Chadwick, N. (2009) 'Determination of ease allowances included in pattern construction methods', International Journal of Fashion Design, Technology and Education, 2(1), pp. 23-31. doi: 10.1080/17543260903018990.

[12] Gill, S. and Parker, C. J. (2017) 'Scan posture definition and hip girth measurement: the impact on clothing design and body scanning', Ergonomics. Taylor \& Francis, 60(8), pp. 1123-1136. doi: 10.1080/00140139.2016.1251621. 